\title{
REDES ARTÍSTICAS Y CULTURALES ALTERNATIVAS EN LA CIUDAD GLOBAL
}

\section{Oihane Sánchez Duro}

Universidad del País Vasco/Euskal Herriko Unibertsitatea. Dpto. Arte y Tecnología. Doctoranda

\section{Resumen}

En el momento en el que la economía de la acumulación entra en crisis, la cultura, y con ella el arte, se enfocan como reactivadores de economías en declive: Io inmaterial pasa a operar como impulsor del cambio, de la transformación urbana y como fuerza expedicionaria para la gentrificación de la ciudad central (Ley 1996, 191). Del mismo modo, se convierte en un elemento indispensable de la industria del ocio y el entretenimiento, fruto de su propia espectacularización. En este sentido surge el proyecto de investigación Bilbao Dé-Tour-Nement. Catálogo de espacios y prácticas artístico-culturales, cuyo objetivo es visibilizar los espacios y la actividad artístico-cultural implícita en la citada ciudad además del circuito más conocido. Dicho trabajo repara en la red de agentes culturales, cuya labor surge de forma cohesionada y comprometida con la cotidianeidad del espacio urbano en el que se sitúan y que habitualmente desarrollan su actividad profesional en condiciones de precariedad.

Palabras-clave: CIUDAD; TURISMO CULTURAL; GENTRIFICACIÓN; CARTOGRAFÍA; ARTISTAS; PRECARIEDAD

\section{ALTERNATIVE ARTISTIC AND CULTURAL NETWORKS IN THE GLOBAL CITY}

\begin{abstract}
At the time when the economy of accumulation suffers a crisis; Culture and Art, are focused as stimulus of economies in decline: the immaterial operates as a promoter of change, of the urban transformation and as expeditionary force for inner- city gentrifiers (Ley 1996, 191). In the same way, it becomes an essential element of the leisure and entertainment industry, fruit of its own spectacularization. It is in this sense that the research project "Bilbao Dé-Tour-Nement. Catalogue of artistic and cultural spaces and practices" comes to light. The aim of the project is to cast light on the implicit artistic and cultural activity and spaces in the aforementioned city, as well as on the primarily known circuit. In the same vein, the project highlights the network of cultural agents who work in this area and whose duty arises in a cohesive manner and is compromised by the everyday life of the urban space in which they find themselves, and who often carry out their professional activity in precarious conditions.
\end{abstract}

Keywords: CITY; CULTURAL TOURISM; GENTRIFICATION; CARTOGRAPHY;ARTISTS; PRECARIOUSNESS

\section{AUSART}




\section{INTRODUCCIÓN}

Presentamos un proyecto que reflexiona en torno a las ciudades que abogan por un modelo productivista de la cultura como un regenerador económico. Tomamos como caso de estudio la ciudad de Bilbao, que como otras ciudades del primer mundo desindustrializadas, se ve en la necesidad de abordar la transición de un modelo económico industrial, a un modelo basado principalmente en el sector servicios.

En la década de los 90 se ponen en marcha diversos proyectos que buscan la regeneración económica de la ciudad, entre los que destaca especialmente la construcción del museo Guggenheim como uno de los elementos del cambio más paradigmáticos. Advertimos claramente una relación recíproca entre cultura y economía como base del capitalismo contemporáneo (Brea 2009). Bien es sabido que a dicho museo, inaugurado en octubre de 1997, se le atribuye haber "colocado la ciudad en el mapa", rompiendo con el pasado convulso de la misma: aún con las secuelas de la crisis del metal de las décadas anteriores, la ciudad no sólo necesitaba recuperarse económicamente, sino que también era necesario reformar la imagen de sí misma a proyectar tanto hacia sus habitantes, como hacia el marco internacional. Bilbao se abría así a la ventana global, posicionándose como una ciudad postmoderna completamente integrada en el panorama universal.

A modo de ejemplo, reparemos en el caso de la ciudad de Málaga, en la cual parece replicarse el llamado "efecto Guggenheim" que describía el profesor y periodista Iñaki Esteban y que ha analizado críticamente el artista Rogelio López Cuenca': esto es, Málaga encuentra en lo simbólico un motor de regeneración económica en torno a un elemento histórico y cultural: la figura del pintor Pablo Picasso, artista de peso incuestionable del siglo XX, nacido en la citada ciudad en 1881. En 2003 se procede a la apertura del Museo Picasso Málaga: una vez más, la institución museo supone una fuerza motriz para la regeneración económica de la ciudad así como polo de atracción turística e inversiones tanto públicas como privadas. Sin embargo, cabe destacar que al contrario que el museo Guggenheim de Bilbao cuya motivación se configuraba en torno a ser "una ventana al arte internacional", el museo Picasso así como otros proyectos e infraestructuras culturales que se dan en Málaga a partir de ese momento serán en torno a la figura de un sujeto-artista que deviene en un sujeto-marca distintiva de la ciudad.

El valor diferencial asociado a los elementos culturales señalados en ambas ciudades, desemboca en la puesta en marcha de políticas y estrategias de creación de una nueva identidad para cada localidad, palpable en ambos casos. Tal y como apostillaba Rubén Martínez de YP Productions², durante su ponencia en el seminario "Repen- 
sando la Metrópolis", que tuvo lugar en Málaga, se trata de la creación de una marca o "branding" que genera áreas de oportunidad de mercado que se alejan de funciones meramente culturales (Esteban 2007, 20-5). De este modo, la construcción de una marca de ciudad capaz de generar el valor distintivo necesario para atraer visitantes y grandes inversiones tanto públicas como privadas, modifica ciertos espacios públicos del tejido urbano, así como las relaciones interpersonales que allí tienen lugar, generando cierta confrontación o dicotomía entre lo global y lo local.

El proyecto de investigación Bilbao Dé-Tour-Nement. Catálogo de Espacios y Prácticas Artístico-Culturales/Espazio eta Praktika Artistiko-Kulturaleen Katalogoa surge con la motivación de recoger y visibilizar la oferta cultural implícita en Bilbao que se desarrolla con independencia del circuito cultural oficial. Por otro lado, subraya múltiples prácticas que se integran en la propia cotidianeidad del entorno social que las motiva, siendo éste un factor fundamental que permite entender el arte no cómo un motor de generación de valor económico, sino como un bien en sí mismo.

\section{A LA SOMBRA DEL MUSEO: CATÁLOGO DE ESPACIOS Y PRÁCTICAS ARTÍSTICO CULTURALES}

Como apostillan Arantza Lauzirika y Natxo Rodríguez (2014),“sin ir demasiado lejos, en la evolución de los procesos de gentrificación, en la que el fenómeno Guggenheim Bilbao se ha convertido en el referente a seguir en el Estado Español y en Europa, las ciudades han optado por dotar al entramado urbanístico de grandes edificios diseñados por arquitectos. Estos reclamos actúan a su vez como reclamos 'escultóricos' y la ciudad va poblándose de contenedores de alto presupuesto en la creencia de que un edificio destinado a la cultura genera cultura per se. La producción artística local, lejos de beneficiarse de la nueva situación, ve cómo estos contenedores se rellenan con exposiciones enlatadas itinerantes que traen grandes masas de visitantes que rentabilizan la inversión. Esta cuestión deriva en un modelo de ciudad, que sustituye los elementos identitarios característicos de cada lugar por otros globalizados que bien podrían pertenecer a otras ciudades de otros países. Estas dinámicas en las políticas culturales macro dejan como legado a las futuras generaciones un modelo artístico y cultural, cuyo efecto homogeneizador no diferencia a oriente de occidente, ni al norte del sur". 
En este escenario surge el proyecto de investigación Bilbao Dé-Tour-Nement. Catálogo de Espacios y Prácticas Artístico-Culturales/Espazio eta Praktika Artistiko-Kulturaleen Katalogoa y cuyo objetivo ha sido el de recoger los espacios e iniciativas artísticas y culturales de Bilbao, activas en la horquilla cronológica comprendida por la duración del proyecto (2015-2016).

Otro de los objetivos del catálogo se ha basado en detectar iniciativas que surgen desde la práctica artística y que cuentan con un marcado componente social que, bien a través de un espacio o sin él, asumen el contexto socio-cultural específico del lugar como parte integral de su práctica y no "como un mero aderezo o complemento de orden sociológico" (Claramonte 2010, 10). Ello resulta relevante pues indica la capacidad del arte y la cultura para integrarse en otras capasque difieren del hecho cultural entendido como un mero producto de exhibición y consumo acotado a una serie de contenedores específicos.

Por citar algunos ejemplos, en 2012, el artista Juan Pablo Ordúñez (MawatreS) pone en marcha un programa de intervenciones artísticas en la caseta del Puente de Deusto ${ }^{3}$ proyecto de comisariado que desarrolla junto a Arantza Lauzirika Morea en colaboración con la facultad de Bellas Artes y el ayuntamiento de Bilbao. Tal y como describen sus organizadores, este proyecto curatorial viene a señalar, por una parte, la recuperación de un elemento arquitectónico del pasado portuario e industrial de la Villa como parte activa de la actividad cultural de la ciudad contemporánea, sin la necesidad de destinar importantes inversiones a macro-equipamientos o proyectos culturales con "apariencia cultural pero con intencionalidad política" (Ordúñez \& Lauzirika2012) o con el ánimo de cumplir con otro tipo de funciones que difieren de la cultural. Por otro lado, se trata de dar visibilidad a un elemento que había quedado en desuso y neutralizado por el entorno y casi imperceptible para los transeúntes a través de una serie de propuestas artísticas cohesionadas con el contexto histórico, social y político del entorno de la caseta. Así, "la caseta se recupera de una forma activa y deja de ser un residuo de la ciudad industrial, para formar parte activa de una ciudad cultural. Su función ahora, es acercar el arte emergente, para mostrarlo en el espacio público de la ciudad de Bilbao, sin que los ciudadanos tengan que visitar salas o galerías, simplemente tienen que pasear para ver",concretan MawatreS y Arantza Lauzirika a propósito de dicho proyecto.

Otro ejemplo de iniciativas culturales coherentes con el entorno son las propuestas desarrolladas por el colectivo Ant Espacio, que trabajan sin un espacio 
de físico estable pero que, sin embargo, elaboran propuestas que se adapten a los diferentes contextos alejándose del marco expositivo habitual. Entre susproyectos, podemos destacar Iturfest ${ }^{4}$ un festival de cultura contemporánea que se realiza en el barrio de Iturralde, en Bilbao. Un pequeño barrio que se pierde rodeado por barrios más grandes. El objetivo radica en que los artistas invitados en cada edición realicen una propuesta específica tanto en espacios públicos como a través de la colaboración con otras instituciones y negocios de la zona, así como contando con la participación de vecinos, asociaciones y colectivos locales. Tal y como define el colectivo Ant Espacio en su página de internet "La idea es redefinir el concepto expositivo en espacios que no estén exclusivamente dedicados al arte. Se organizarán diferentes tipos de actividades en los diferentes espacios propuestos y los artistas invitados realizan proyectos específicos para el espacio y el tiempo. La "experiencia artística" nace ante todo, de la temporalidad del acontecimiento y del encuentro casual. Nuestro objetivo es intervenir por medio del arte en el espacio cotidiano y los artistas logran que el espacio cotidiano sea visto desde otra perspectiva".

Mediante estos dos ejemplos. Podemos distinguir propuestas que parten del arte y la cultura en las que "se opta por procesos de distribución en esferas públicas menos segregadas socialmente que las del mundo del arte, y que pueden oscilar entren las pequeñas comunidades vecinales y los grandes medios de comunicación y de relaciones sociales y políticas, siendo este proceso de distribución y recepción no un mero residuo de la productividad artística, sino un factor central para su comprensión y su retroalimentación" (Claramonte 2010, 16).

\subsection{DESCRIPCión DEL PROYECTO Y METOdOLOGía}

Durante la realización del proyecto se entrevistaron 55 agentes provenientes de distintos ámbitos del arte y la cultura, haciendo especial hincapié en la relación de la actividad que desarrollan con el contexto en el que se ubica, así como en los vínculos entre otros agentes, actividades y espacios. Se genera de este modo una red relacional en torno a intereses afines, transformando continuamente sus actividades, buscando nuevas fórmulas en base a las que trabajar partiendo lo social, lo colaborativo y participativo, hibridando distintos formatos y personas.

Se presentan perfiles propios de asociaciones culturales, cooperativas, colectivos, intervenciones y proyectos artísticos de carácter efímero o de periodicidad concreta, artistas individuales, estudios compartidos, galerías, salas 
expositivas institucionales o independientes, museos de distinta categoría, espacios polivalentes, fundaciones, etc. pertenecientes a cualquier disciplina o materialización contemporánea del quehacer artístico.

De este modo, el catálogo se compone de dos libros relacionados entre sí, acompañados por un mapa que recoge los espacios en activo durante su realización:

1. (1) Quién/Qué-Nork/Zer: un libro dedicado especialmente a los agentes y equipos de personas que llevan a cabo las distintas iniciativas artísticas y culturales, incluyendo una breve descripción sobre su trayectoria formativa y profesional en dicho ámbito.

2. (2) Dónde/Cómo-Non/Nola: recoge los espacios y aquellos lugares en el que se llevan a cabo las actividades y todo tipo de expresiones artístico-culturales, así como la metodología que hace posible su puesta en marcha.

3. (3) El mapa recopila las localizaciones de aquellos agentes entrevistados y espacios visitados.

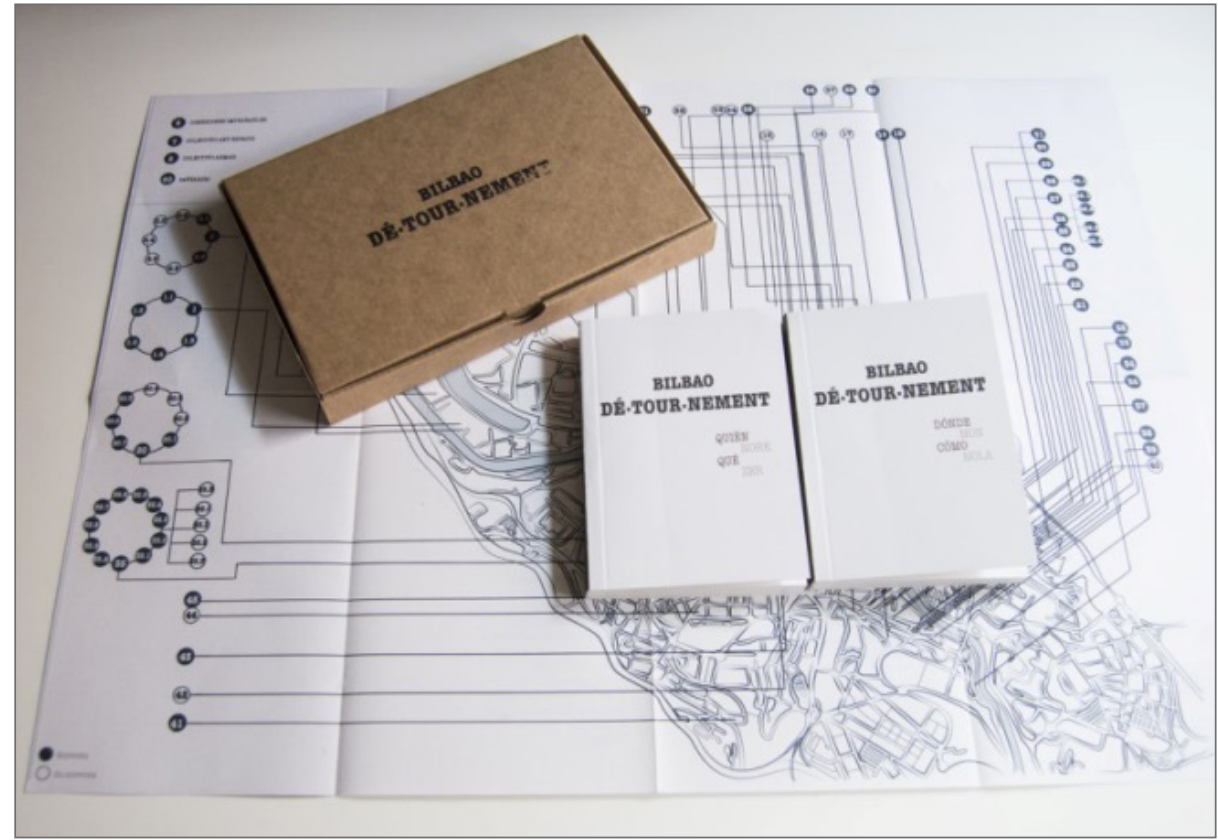

Fig. 1: el catálogo y sus elementos 


\subsubsection{Desarrollo del proyecto a través de sus distintas FOrma- LIZACIONES}

Atendiendo a la cuestión que aborda el presente documento (¿cómo se cuentan las cosas?) en primer lugar debemos señalar que el proyecto recientemente descrito es un estudio que toma lo artístico como punto de partida metodológico. El mismo se inicia motivado por la acción del détournement, concepto surgido dentro del movimiento situacionista ${ }^{5}$ que habla sobre la posibilidad artística y política de tomar algún objeto creado por el capitalismo o el sistema político hegemónico y distorsionar su significado y uso original para producir un efecto crítico.

Conforme la cultura "se convierte en reactivador de economías en declive" (Lauzirika \& Rodríguez 2014, 43), afloran las guías y planos turísticos oficiales que recogen los equipamientos culturales más emblemáticos de la ciudad, sobreentendiendo la cultura como un producto de consumo espectacularizado, como apostillaba en 1967el teórico situacionista Guy Debord en su obra La sociedad del espectáculo y en la línea de Lauzirika y Rodríguez,que aducen que un contenedor cultural, convertido en un elemento emblemático insertado en el paisaje urbano, no necesariamente genera cultura ni recursos de los cuales pueda beneficiarse el tejido de artistas locales.

Por ello, se remite al concepto del détournement, a fin de visibilizar esa otra oferta cultural implícita en la ciudad, recogiendo una mayor diversidad de iniciativas y expresiones artístico-culturales heterogéneas, que operan con mayor cohesión en relación al contexto urbano en el que emergen y se desarrollan.

Desde este momento en el que acción artística y trabajo de campo se unen, tanto el desarrollo conceptual como el formal operarán en conjunto asentando las bases desde las que partirá la evolución de las distintas formalizaciones del proyecto Bilbao Dé-Tour-Nement. Catálogo de Espacios y Prácticas Artístico-Culturales. Las exposiciones que se describen hablan del declive del modelo precedente y del paso a una economía postfordista en las que lo inmaterial pasa a ocupar un lugar central en relación a las nuevas formas de producción y consumo. Uno de los referentes en torno a este eje es el artista británico Jeremy Deller, cuyo trabajo se vertebra en torno al cambio de Reino Unido hacia una sociedad postindustrial ${ }^{6}$. Sus obras incluyen, precisamente, una clara reflexión en torno al desplazamiento social mismo mediante el cual la sociedad pasa de ser productora a ser consumidora como consecuencia implícita del tránsito hacia el capitalismo tardío (Ferrero 2010). En lo que concierne al proyecto Bilbao Dé-Tour-Nement. Catálogo de Espacios y Prácticas 
Artístico-Culturales,se establece un análisis desde el arte en torno al tránsito de un modelo de ciudad a otro, el cual constatamos en la transformación de espacios industriales obsoletos en espacios para el desarrollo de iniciativas y prácticas artístico-culturales heterogéneas que además resultan un interesante objeto de estudio por situarse, en muchos casos, al margen de los espacios y circuitos legitimados para el arte en la ciudad contemporánea.

Las diferentes formas de presentación que va adquiriendo el proyecto profundizan paulatinamente en la investigación artística interdisciplinar hibridando distintas inquietudes dentro de sus posibilidades plásticas a través de formatos estrechamente ligados a la instalación y que paulatinamente se expanden a través de la inclusión de cartografías o mapas relacionales que permiten un mayor análisis sobre las características del contexto socio-cultural que envuelve el trabajo mencionado.

\subsubsection{La instalación como extensión del formato impreso}

Desde la presentación de la publicación en papel del proyecto Bilbao Dé-Tour-Nement. Catálogo de Espacios y Prácticas Artístico-Culturales en julio de 2016, este se ha dado a conocer en varias ocasiones mediante el formato expositivo (entre otras, en la Fundación Bilbaoarte; en la Sala Rekalde y durante la exposición Creative Room, actividad que formaba parte del tercer congreso internacional Arte, Ciencia, Ciudad, que tuvo lugar en la Universidad de Málaga). Las muestras enumeradas han tenido como fin indagar, como se comentaba en el párrafo anterior, en el marco socio-cultural concreto en el que se inscribe el proyecto: partiendo del catálogo se despliegan formas poéticas de visualización de los contenidos inherentes a la dicotomía entre dos modelos diferentes de ciudad, hecho que ha motivado, precisamente, recoger y visibilizar los otros modos de hacer propios del ámbito artístico-cultural pero que sin embargo divergen del modelo hegemónico de ciudad cultural y de servicios.

En un primer estadio, la muestra que tuvo lugar en la Fundación Bilbaoarte del 9 al 16 de septiembre de 2016 trata de aunar los dos aspectos ahora comentados: A consecuencia de la fuerte desindustrialización que experimenta Bilbao a finales de los años 80 , pabellones, edificios, fábricas y naves industriales en desuso pasan a configurarse como una alternativa para los creadores: espacios diáfanos, luminosos y versátiles, que bien porque el lugar constituye el propio leitmotiv de estas prácticas o bien porque el lugar mismo brinda alternativas concretas de trabajo, constituyen espacios de ensayo, de creación, de 
intervención, de exhibición y a la vez suponen lugares de encuentro e intercambio para la comunidad artística y cultural local.

Con el objetivo de trasladar el contraste existente entre una actividad basada en los modos de producción industrial a los modos de producción propios de la era del postfordismo,la instalación alude, por una parte, a los espacios en desuso pertenecientes a los modos de producción ligados a un estado anterior, y que por otro lado enfatizan al mismo tiempo otro tipo de morfologías desde el quehacer de la práctica artística que diverge de los formatos y lugares convencionales para la creación, la difusión y el consumo de arte y cultura.
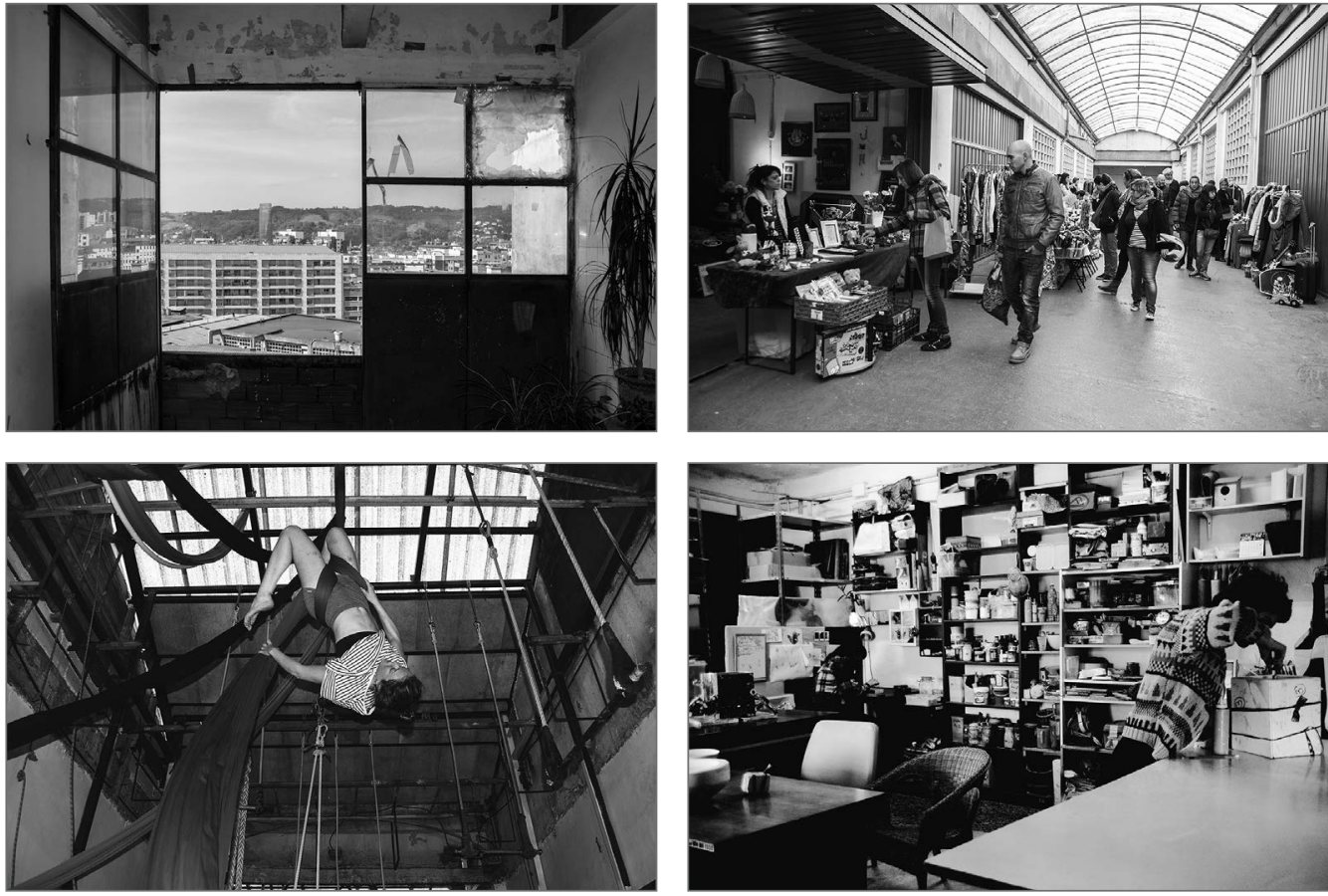

Figs. 2,3,4 y 5: fotografías extraídas del catálogo

Aunque la metamorfosis de muchos edificios industriales en espacios para el arte y la cultura es algo recurrente a lo largo de la ciudad y especialmente en barrios periféricos o en proceso de gentrificación, encontramos un ejemplo paradigmático en Zorrotzaurre,una zona industrial resituada como lugar de interés en el proceso de ampliación de la ciudad de Bilbao, cuyos edificios y fábricas han enfrentado durante décadas un largo proceso de deterioro fruto 
del abandono y que hoy por hoy se encuentra en pleno proceso de transformación urbana ${ }^{7}$.

A raíz del derrumbe de unas oficinas de una planta de fabricación de materiales abrasivos en julio de 2016 se recuperan objetos que pasan a formar parte de la instalación realizada en Bilbaoarte. De este modo, las series de fotografías y materiales se superponen como si de estratos o capas se trataran: la ciudad vieja, condensada en esos objetos y espacios ligados a los modos de producción tangibles; y la ciudad contemporánea, en relación a esos nuevos modos de producción simbólica.

Los ejemplares del catálogo Bilbao Dé-Tour-Nement. Catálogo de Espacios y Prácticas Artístico-Culturales, se colocaron en el centro de la sala a disposición de todas aquellas personas interesadas en el mismo durante todo el tiempo de exposición. Cabe destacar que, aun estando disponible para los visitantes, éste no es el catálogo al uso que encontramos en las exposiciones, sino que ha servido como elemento de partida para articular un dispositivo visual en formato de la instalación: lo que el visitante puede llevarse consigo a través del catálogo es una suma de nuevos modos de hacer (de alternativas) inscritos en el cambio de un modelo de ciudad a otro.
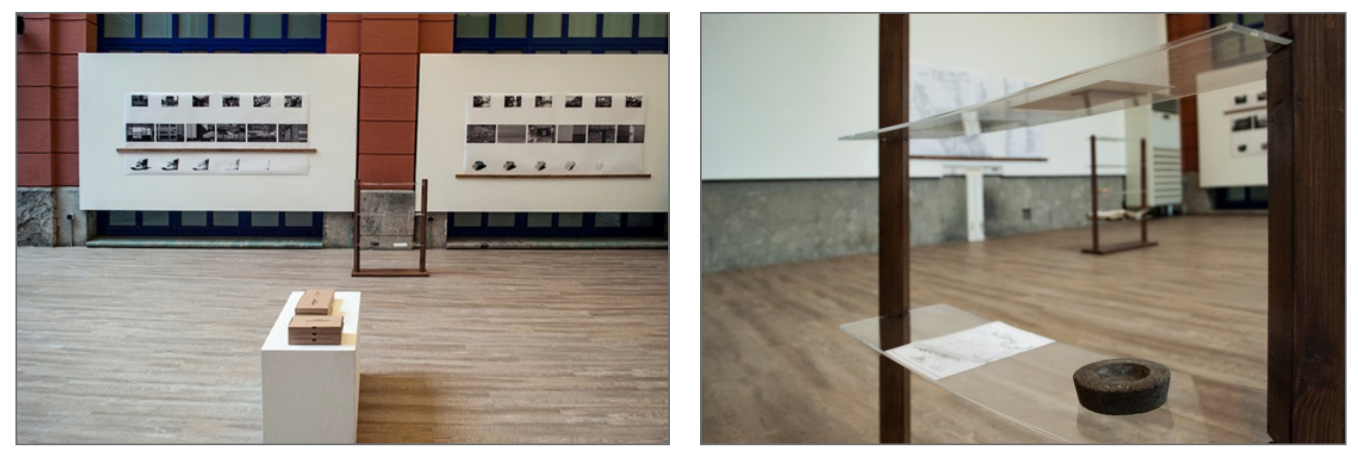

Figs. 6 y 7: Détournement, 2016

2.1.1.2. Redes: Expandiendo los límites a través de los mapas relacionales

La muestra realizada pocos meses después en la Sala Rekalde Aretoa, del 16 de febrero al 6 de marzo de 2017, continúa con ese carácter propio del formato de la instalación en el que las fotografías y el material de archivo mantienen el contraste descrito antes en relación a los usos y posibilidades de los espacios, la tensión existente entre la ciudad pasada y la ciudad contemporánea. 
Para esta ocasión se realiza un vídeo en el que se invita a artistas y gestores culturales locales a reflexionar sobre conceptos y temáticas inherentes al marco discursivo que envuelve el proyecto Bilbao Dé-Tour-Nement, a fin de generar un diálogo común mediante las distintas conversaciones (aunque afines entre ellas). Los temas tratados fueron el contexto, lo cotidiano, el margen y la periferia, la memoria y la conmemoración.

Los diálogos generados en el vídeo conforman un primer intento por expandir las limitaciones del formato de la publicación como la forma original que adquiere el proyecto que se describe. Entre otras, la propia obsolescencia del formato impreso en relación a un ámbito tan dinámico y cambiante como es el cultural: frecuentemente, aparecen nuevos espacios y nuevas iniciativas, otras modifican su actividad, su lugar de trabajo o su ubicación dentro de la ciudad, sus miembros y participantes, actividades que cesan y desaparecen.

\subsubsection{Otras presentaciones: Creative room}

Siguiendo este hilo, las últimas materializaciones destacan por una actuación directa sobre el propio plano que acompaña los libros que conforman el catálogo y que ira adquiriendo un mayor protagonismo en las siguientes presentaciones: tomando éste como herramienta se propone intervenir de forma colectiva sobre el mismo generando grupalmente los mapas posibles inscritos en el actual, a fin de obtener una suma de visiones y sensibilidades que enriqueciera el contenido mostrado hasta la fecha. Este fue el objetivo del taller impartido el 3 de mayo en Sarean, espacio cultural y de encuentro vecinal situado en la calle San Francisco de Bilbao. Durante el taller, se les pidió a los asistentes tomar parte en el mapa desde sus propias referencias y relaciones, sumando espacios, personas y actividades, así como reflexiones y connotaciones acerca de los diferentes territorios urbanos, incluyéndose finalmente a sí mismos en una red que los vinculaba con el conjunto a través de los diferentes elementos volcados.

El resultado del taller es expuesto en la actividad Creative Room dentro del 3er Congreso Internacional sobre Arte, Ciencia y Ciudad organizado por la Universidad de Málaga en noviembre de 2017: a través de la intervención colectiva comentada, se plantea una aproximación a esa extensa amalgama de redes artísticas y culturales alternativas que habitualmente se conforman con independencia de los circuitos culturales más normativizados de la ciudad global. 

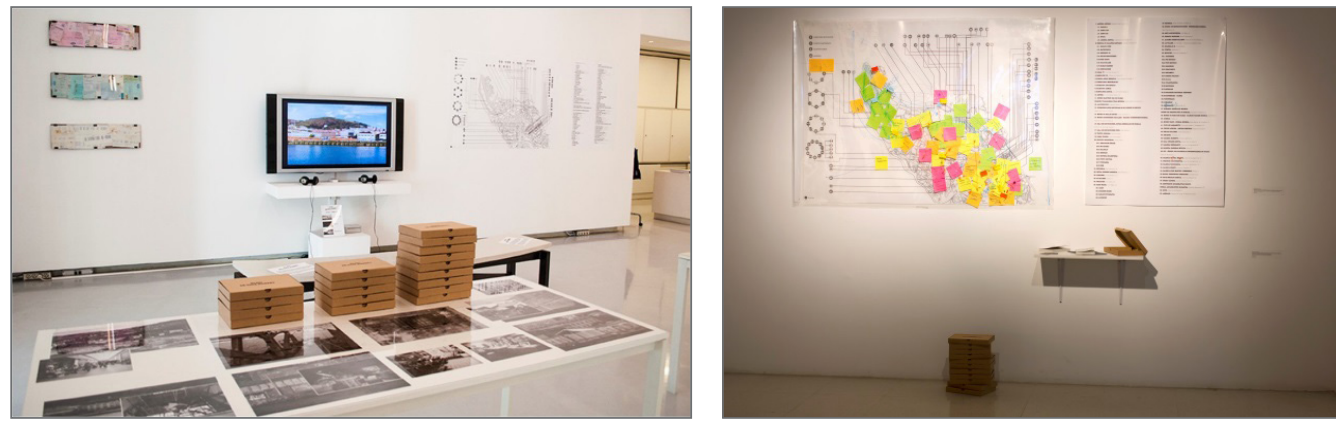

Fig. 8 y 9: Bilbao Détournement, 2017. Arte, Ciencia, Ciudad: Creative Room (fotografía de Fran Carneros), 2017.

\subsubsection{Proyecto de investigación Mekarteak}

Paralelamente, se desarrolla un trabajo en colaboración con el comisario IkerFidalgo Alday para el proyecto de investigación Mekarteak: Meta-cartografías del arte en el País Vasco/Euskal Herriko artearen meta-kartografiak impulsado desde la facultad de Bellas Artes de la UPV/EHU. Los objetivos de Mekarteak convergen en romper las clasificaciones hegemónicas desde las cuales se trata de dar cuenta de dicho ámbito a través de la revisión, inclusión y ampliación de nuevos mapas. Podemos deducir que el trabajo de investigación realizado por el grupo genera una serie de contra-mapas (Sánchez 2017) que ofrecen una pluralidad de visiones contrapuestas a la conocida cartografía sobre el arte en el País Vasco elaborada por el artista y profesor Juan Luis Moraza ${ }^{8}$

La última exposición organizada en el edificio Bizkaia Aretoa de la UPV/EHU en octubre de 2017 por el equipo investigador, aludía precisamente a la necesidad de huir de las clasificaciones habituales pues "el terreno es dinámico y no tiene límites claros; tiene multitud de capas complejas, entremezcladas e interconectadas que la representación ortogonal no consigue atrapar" (Mekarteak 2017).

Por ejemplo,en nuestra aportación realizada junto con Iker Fidalgo, tratamos de hacer hincapié en las múltiples redes que unen los distintos elementos permitiendo expandir las categorías estancas bajo las que normalmente trata de analizarse un ámbito tan heterogéneo y difuso como el artístico y el cultural. Tal y como se recoge en la presentación ${ }^{8}$ incluida en la caja-objeto que reúne los materiales de la muestra e investigación:"De un lado, Iker Fidalgo plantea algunas reflexiones sobre los cambios acontecidos desde la publicación del referencial mapa de Juan Luis Moraza para el Guggenheim. A partir de ahí, 
(Iker Fidalgo) propone para un "contexto postcrisis": "tachar lo que desaparece". Más adelante se opta por una revisión de la idea de constelación y, por tanto, de la necesidad de nuevas formas de visualizar las relaciones en el contexto local del arte y la cultura. Así, desde este planteamiento se procede a la revisión del panorama cultural a través de nuevos horizontes de prácticas culturales instituyentes" (Mekarteak 2017).

\section{CONCLUSIONES}

Especialmente en la primera etapa de este proyecto durante la cual se publica el catálogo Bilbao Dé-Tour-Nement. Catálogo de Espacios y Prácticas Artístico-Culturales, la motivación artística opera junto a estrategias propias del desarrollo del trabajo de campo, basado en búsqueda de información, recopilación de documentación audiovisual y realización de entrevistas fundamentalmente. Ello hace denotar cierto peso sociológico en torno al proceso.

No obstante, y recordando que se parte de una inquietud artística como base, el lenguaje del formato de la instalación permite abordar el proyecto desde un punto de vista plástico, tanto en lo relativo a la forma como al tratamiento de los contenidos. La publicación, en diálogo con el resto de materiales, pasa a formar parte del conjunto, constatando que aún supone el eje central y por ello mismo este es el producto que los visitantes podían llevarse durante las diferentes muestras llevadas a cabo.

Si atendemos la evolución general del proyecto a lo largo de sus distintas presentaciones, comprobamos que ha habido un movimiento desde el objeto hacia estrategias que permiten la integración del contexto que se analiza, y que gradualmente comienza a adquirir un notable carácter y formato en red, en tanto que el proyecto se abre, y requiere la participación de otras personas tal y como vemos durante la Intervención colectiva sobre el mapa (proceso que se muestra en la actividad Creative Room, paralela al congreso Arte, Ciencia, Ciudad mencionado anteriormente) donde se buscaba la participación activa a través del encuentro o la citación dentro de una propuesta concreta y cuyo objetivo era apelar a los mapas relacionales de los propios participantes incorporándolos inmediatamente al grueso del proyecto. Finalmente, la propuesta se abre a otros formatos y contenidos en la colaboración con el comisario lker Fidalgo Alday que acabamos de describir. 
El objetivo del desarrollo de las distintas posibilidades plásticas del proyecto no sólo versaría en ofrecer una aproximación poética y crítica sobre el contexto socio-cultural que nos ocupa, sino que al mismo tiempo quiere elaborar un dispositivo en continua revisión y actualización que dé cuenta de la capacidad de generatividad y desarrollo de los espacios y prácticas artístico-culturales en Bilbao, así como de las múltiples capas de relación entre cada elemento mediante los lazos que conectan personas, espacios, actividades y su cohesión en relación a distintos territorios urbanos.

En definitiva y apoyada por las estrategias y lenguajes propios del arte, se trata de rescatar la lectura al margen del discurso dominante, reclamando la presencia y riqueza de las diversas expresiones artísticas y culturales en el mismo, que con independencia de estos contenedores-marca, realizan una aportación constante en el ámbito artístico-cultural de Bilbao, y que sin embargo ni son lo suficientemente visibilizadas, ni muchas las garantías para su perdurabilidad.

\section{Referencias}

Brea, José Luis 2009. El tercer umbral. Estatuto de las prácticas artísticas en las sociedades del capitalismo cultural. Murcia: Cendeac

Esteban Azurmendi, Iñaki. 2007. El Efecto Guggenheim: Del espacio basura al ornamento. Barcelona: Anagrama

Claramonte Arrufat, Jordi.2010. Arte de contexto. Donostia-San Sebastián: Nerea

Debord, Guy .(1967) 2002. La sociedad del espectáculo. Prólogo, traducción y notas de José Luis Pardo. Valencia: Pre-Textos

Ferrero Brotons, Ángel. 2010. "La Batalla de Orgreave: Entre el simulacro, el teatro de masas y el cine político". Nómadas 25(1):127-49. https://doi.org/10.5209/NOMA.27089

Lauzirika Morea, Arantza \& Natxo Rodríguez Arkaute. 2014. "Arte y trabajo”. En Áreas emergentes e innovación en el sector cultural y creativo vasco, eds., Aihnoa Estankona, Arantza Lauzirika y Natxo Rodríguez, 41-51. Bilbao: Universidad del País Vasco

Ley, David.1996. The new middle classes and remaking of central city Oxford: Oxford University

Mekarteak: Euskal Herriko artearen meta-kartografiak [Meta-cartografías del arte en el País Vasco].2017. 14 obras multiformato (pósters, fanzines, chapas, pegatinas, mapas...), versión portátil de las exhibidas en Bizkaia Aretoa, 17-20 oct. Bilbao: Universidad del País Vasco

Ordúñez Martínez, Juan Pablo \& Arantza Lauzirika Morea. 2012."Arte para recuperar la memoria". Arte Puente de Deusto (blog), 27 dic. http://artepuentededeusto.blogspot.com/2012/12/ arte-para-recuperar-la-memoria.html?q=Arte+para+recuperar 
Sánchez Duro, Oihane. 2017. "Contra-mapas: El imaginario cartográfico como acción". En Contra escritos sobre crítica \& contracultura, Koldo Abad et al., 117-126. Bilbao: Popurrit

\section{Notas}

${ }^{1}$ Surviving Picasso es un proyecto de investigación y creación artística en curso que analiza precisamente el hecho descrito en torno a la figura de Pablo Picasso y su relación con Málaga. Este proyecto señala la transformación del centro histórico de Málaga en un parque temático sobre la vida y obra del pintor, y que ha devenido en un producto de consumo atractivo para el turismo cultural en masa. http://www.malagana.com/surviving_picasso/ intro.html

${ }^{2}$ Rubén Martínez responsable de YP Productions, empresa de servicios culturales que contribuye a la creación de procomunes.

${ }^{3}$ http://artepuentededeusto.blogspot.com.es/

${ }^{4}$ https://antespacio.com/espacios/festival-iturfest/

${ }^{5}$ El movimiento situacionista o situacionismo sería la denominación del pensamiento y la práctica en la política y las artes inspirada por la Internacional Situacionista (1957-1972) configurada por varios grupos vanguardistas del momento.

${ }^{6}$ Una de las obras más características que sintetiza este cambio es la recreación de la batalla minera de Orgreave, hecho histórico que tuvo lugar en 1984 cuando 1.800 mineros se enfrentaron a 1.500 policías en esta localidad de South Yorkshire, en Reino Unido como respuesta al cierre de las minas (en el caso concreto de Orgreave, frente al desmantelamiento de una planta de coque).

${ }^{7}$ El Plan General de Ordenación Urbana de Bilbao de 1995 adelantaba el cambio de uso (de industrial a residencial) de la península de Zorrotzaurre. La transformación de la misma en isla es parte del proyecto Master Plan elaborado por la arquitecta Zaha Hadid en 2004. Ver http://www.zorrotzaurre.com/proyecto-regeneracion-urbanistica-de-zorrotzaurre/ [Acceso24 feb. 2018].

${ }^{8}$ Incógnitas. Cartografías del arte contemporáneo en Euskadi se trata de una obra realizada por Juan Luis Moraza en 2007 para el museo Guggenheim de Bilbao. Para su realización, el autor difundió un cuestionario entre varias generaciones de artistas a fin de obtener los datos que conforman el mapa. https://www.guggenheim-bilbao.eus/exposiciones/incognitas-cartografias-del-arte-contemporaneo-en-euskadi/

${ }^{9}$ El texto para la presentación de la colaboración compuesta por Iker Fidalgo Alday y Oihane Sánchez Duro lo realiza Arturo/Fito Rodríguez Bornaetxea, integrante del equipo de investigación. 